\title{
Orobanchen, verkannte Schönheiten
}

\author{
Holger Uhlich
}

\begin{abstract}
Species of the broomrape family (Orobanchaceae) are parasitic plants. Locally they can damage agricultural plants. Species found in Hesse are shortly described.
\end{abstract}

\section{Zusammenfassung}

Sommerwurz-Arten (Orobanche, Phelipanche) sind Vollparasiten, die lokal auch Schäden in der Landwirtschaft anrichten können. Einige parasitieren nur ganz bestimme Arten. Die in Hessen vorkommenden Sommerwurz-Arten werden kurz vorgestellt. Einen schöner Bestand von Phelipanche ramosa existiert im Botanischen Garten Frankfurt

\section{Sommerwurz im Botanischen Garten Frankfurt}

Sie tut es! Schon wieder! Jedes Jahr verwandelt sich von Ende Juli bis Anfang August die ansonsten nackte Erde unter den Hanf- und Tomatenpflanzen im Gewächshaus des Botanischen Gartens Frankfurt in ein Blumenbeet. Hunderte von Blütenstängeln der Ästigen Sommerwurz (Phelipanche ramosa, früher Orobanche ramosa) durchbrechen die Erdoberfläche und entfalten ihre kleinen, bis $17 \mathrm{~mm}$ langen, blauvioletten Blüten.

Was den Botaniker freut, ärgert den Landwirt und kann in einigen, von der Landwirtschaft stärker abhängigen Gegenden der Erde sogar Panik auslösen. Denn die Sommerwurz ist ein Vollschmarotzer und befällt andere Pflanzen, vorwiegend Wild-, aber auch Kulturpflanzen.

\section{Sommerwurz als Parasit}

Nur wenige der etwa 200 Arten der Gattungen Orobanche und Phelipanche beeinträchtigen in nennenswertem Maße Kulturpflanzen: So parasitieren vor allem Arten wie Phelipanche ramosa und Phelipanche aegyptiaca auf Nachtschattengewächsen (z. B. Kartoffel, Tomate, Aubergine und Tabak), Orobanche cumana auf Sonnenblume, O. crenata auf Puffbohne und O. minor auf Klee. Der weit überwiegende Teil der Sommerwurz-Arten jedoch ist in dieser Be-

Abb. 1: Phelipanche ramosa im Botanischen Garten Frankfurt am Main (9.8.2011).

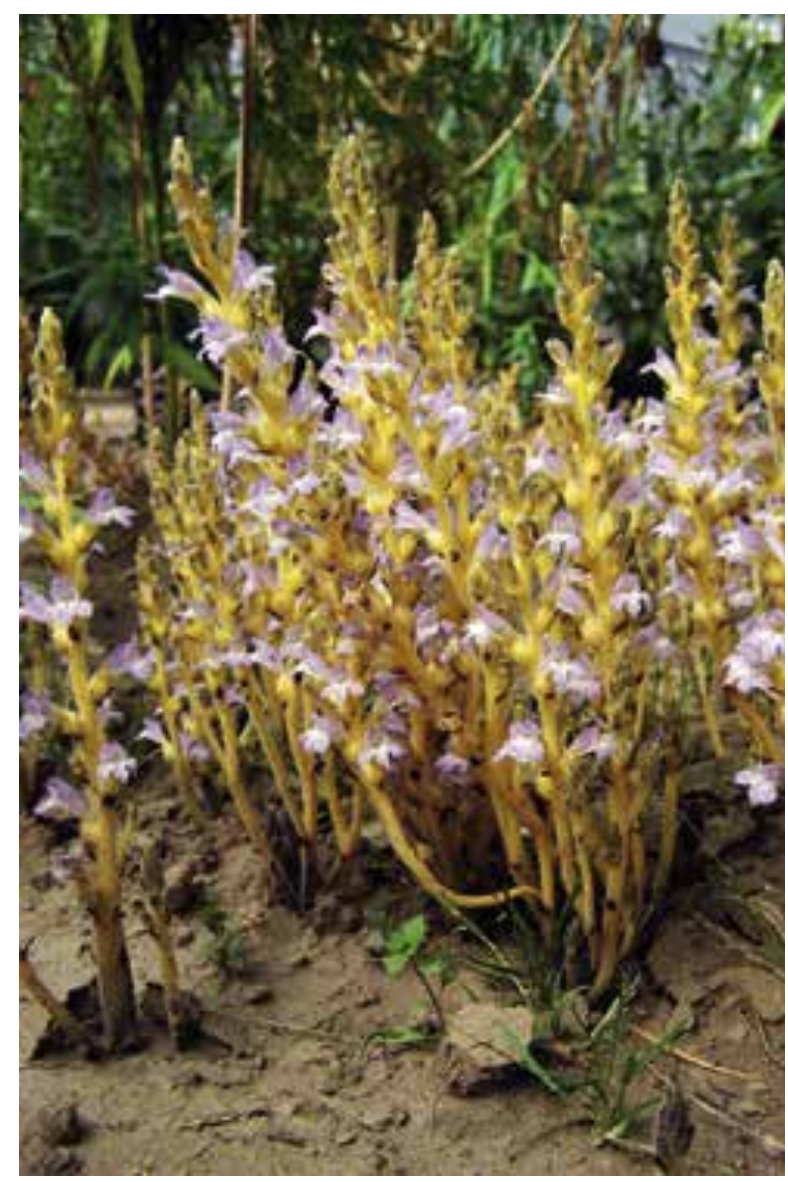

ziehung unauffällig, meist nur auf Wildpflanzen anzutreffen und überdies recht selten. Sie sind, wie Orchideen, schützenswerte Besonderheiten der Flora.

Moderne phylogenetische Untersuchungen haben gezeigt, dass die Familie der Sommerwurzgewächse (Orobanchaceae), also nicht nur die beiden o. g. Gattungen, in verwandtschaftlicher Hinsicht viel weiter zu fassen ist. Mittlerweile zählen daher zur Familie der Oroban- 


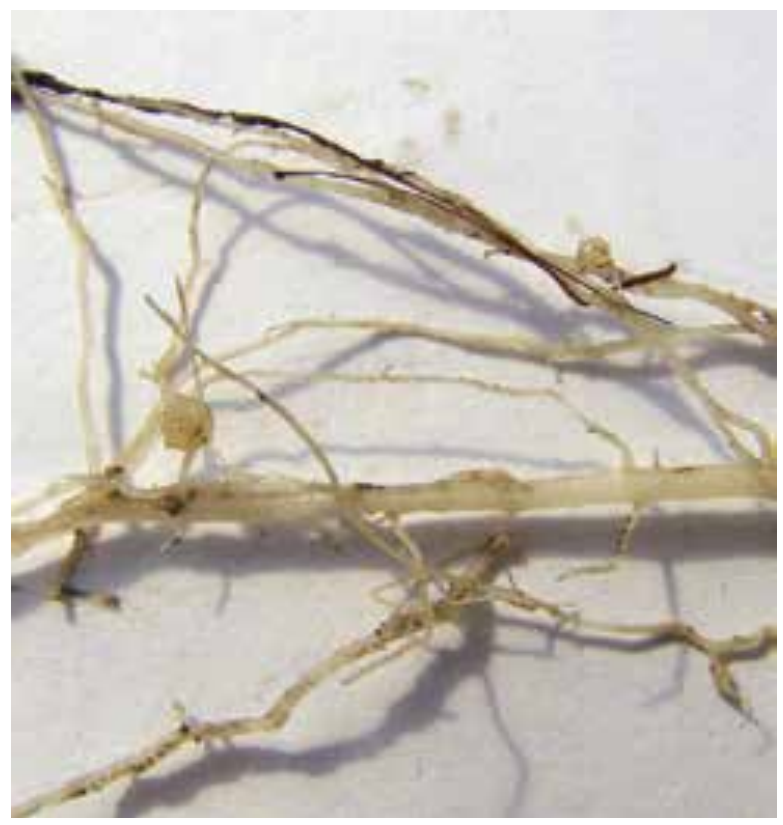

chaceae auch viele Halbparasiten wie z.B. Klappertopf (Rhinanthus), Augentrost (Euphrasia), Läusekraut (Pedicularis), Wachtelweizen (Melampyrum), die früher zu den Rachenblütlern gezählt wurden. Die Orobanchaceae umfassen aktuell etwa 2100 Arten in 96 Gattungen.

\section{Standorte und Verbreitung der Sommerwurz}

Während in Europa ungefähr 70 Arten der Gattungen Orobanche und Phelipanche wachsen, sind es in Deutschland 22 bis 23. Wir finden diese Arten vorwiegend in offenen, wärmegetönten Lebensräumen wie z. B. in Steppen-, Trocken- und Halbtrockenrasen, an Waldrändern und in Gebüschsäumen. Einige wenige Arten bevorzugen jedoch auch schattige Waldstandorte wie z. B. Efeu-Sommerwurz (Orobanche hederae), Salbei-Sommerwurz (Orobanche salviae) und Hain-Sommerwurz (Orobanche lucorum).

Betrachtet man die weltweite Verbreitung der beiden Gattungen Orobanche und Phelipanche, fällt eine Konzentration auf die temperaten Bereiche der Nordhalbkugel auf. Daneben gibt es lokale Vorkommen einiger Punkt-Endemiten im Bereich der Anden Südamerikas (Orobanche chilensis, O. weberbaueri, O. tarapacana, O. tacnaensis) sowie, durch den Menschen bedingte, Vorkommen im tropi-

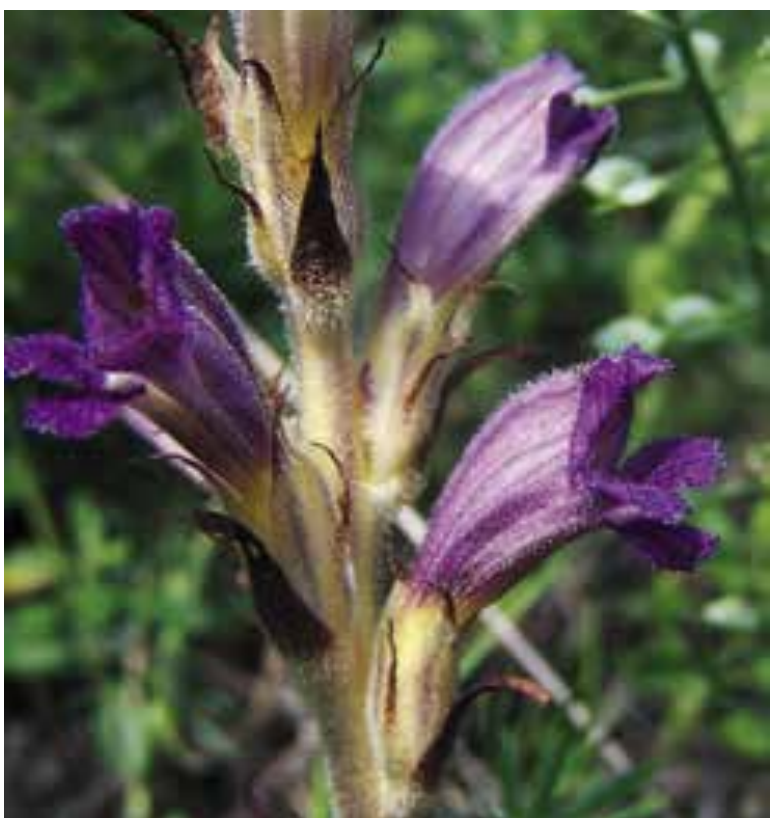

schen und südlichen Afrika, in Südamerika sowie Australien (Orobanche minor, O. cernua, Phelipanche ramosa). Bei den verschleppten Arten handelt es sich zumeist um Arten, die Kulturpflanzen befallen und mit Saatgut eingeschleppt wurden. Süd- und Südostasien ist eher die Heimat verwandter Gattungen wie z. B. Aeginetia, Christisonia u.a. In der borealen Zone gibt es eine weitere nahestehende Gattung (Boschniakia).

\section{Systematik}

In Abhängigkeit des jeweils vertretenen taxonomischen Konzepts zerfallen die beiden Gattungen in insgesamt fünf Sektionen:

Die Gattung Phelipanche, 1874 von Pomel beschrieben, wurde und wird von einem Teil der Botaniker nicht als eigenständige Gattung akzeptiert und als Sektion Trionychon der Gattung Orobanche angesehen. Maßgebliche morphologische Unterschiede (Bau des Kelches, Vorhandensein von Vorblättern, Gestalt der Blüte, Neigung des Stängels zur Ästigkeit) und andere Unterschiede (diploider Chromosomen-

Abb. 2 (links): Frühes Entwicklungsstadium von Phelipanche ramosa auf Hanf (Cannabis sativa) im Botanischen Garten Frankfurt am Main (18.5.2012).

Abb. 3 (rechts): Phelipanche arenaria f. ionantha, Nahetal, Schlossböckelheim (14.6.2010). 
satz von $2 \mathrm{n}=24$ statt wie bei Orobanche i. e. S. $2 n=38)$ sprechen für eine Eigenständigkeit der Gattung Phelipanche. Unter Beachtung der Diversitätszentren dürfte sie ihren Ursprung im westlichen Mittelmeergebiet und in einem kleinasiatisch-kaukasisch-westasiatischen Areal haben. In letzter Zeit wurden vor allem in Spanien einige neue Arten beschrieben.

Die Gattung Orobanche i. e. S. zerfällt in vier Sektionen: Gymnocaulis, Myzorrhiza, Inflatae und Orobanche. Die ausschließlich auf Nordamerika beschränkte Sektion Gymnocaulis besteht aus 2 bis 3 Arten und ist im eher humiden zentralen und atlantischen Gebiet der USA und Kanadas verbreitet, kommt jedoch auch bis zur Pazifikküste vor.

Die ebenfalls nur in Nordamerika und Mexiko vorkommende Sektion Myzorrhiza umfasst etwa 17 Arten auf meist trockenen, ariden Standorten. Allein Orobanche ludoviciana und die erst 2009 beschriebene Orobanche riparia strahlen weiter nach Osten bis in die zentrale und südöstliche USA aus. Diese Sektion hat ihren Ursprung mit ziemlicher Eindeutigkeit in Kalifornien und dem Südwesten der USA.

In der Sektion Inflatae gibt es etwa 20, vorwiegend in Asien beheimatete Arten, die sich von der folgenden Sektion vor allem durch die über dem Fruchtknoten mehr oder weniger deutlich eingeschnürte Kronröhre unterscheiden. Hierzu zählen in Europa Orobanche coerulescens und einige Arten aus der Orobanchecernua-Gruppe (O. cernua, O. cumana und O. grenieri). Auch die (auch im Palmengarten blühende) Orobanche hederae gehört zu dieser Sektion.

Die Sektion Orobanche umfasst den Hauptteil aller Arten der Gattung und kommt natürlicherweise in Europa, N-Afrika, Asien bis nach China und im Himalaya vor. Sie ist ebenfalls an der fernöstlichen Pazifikküste und auf den vorgelagerten Inseln sowie in Japan heimisch. In

Abb. 4: Bergkümmel-Sommerwurz (Orobanche laserpitii-sileris) auf dem Montagne des Mémises (Savoyen, Schweiz, 16.7.2012).

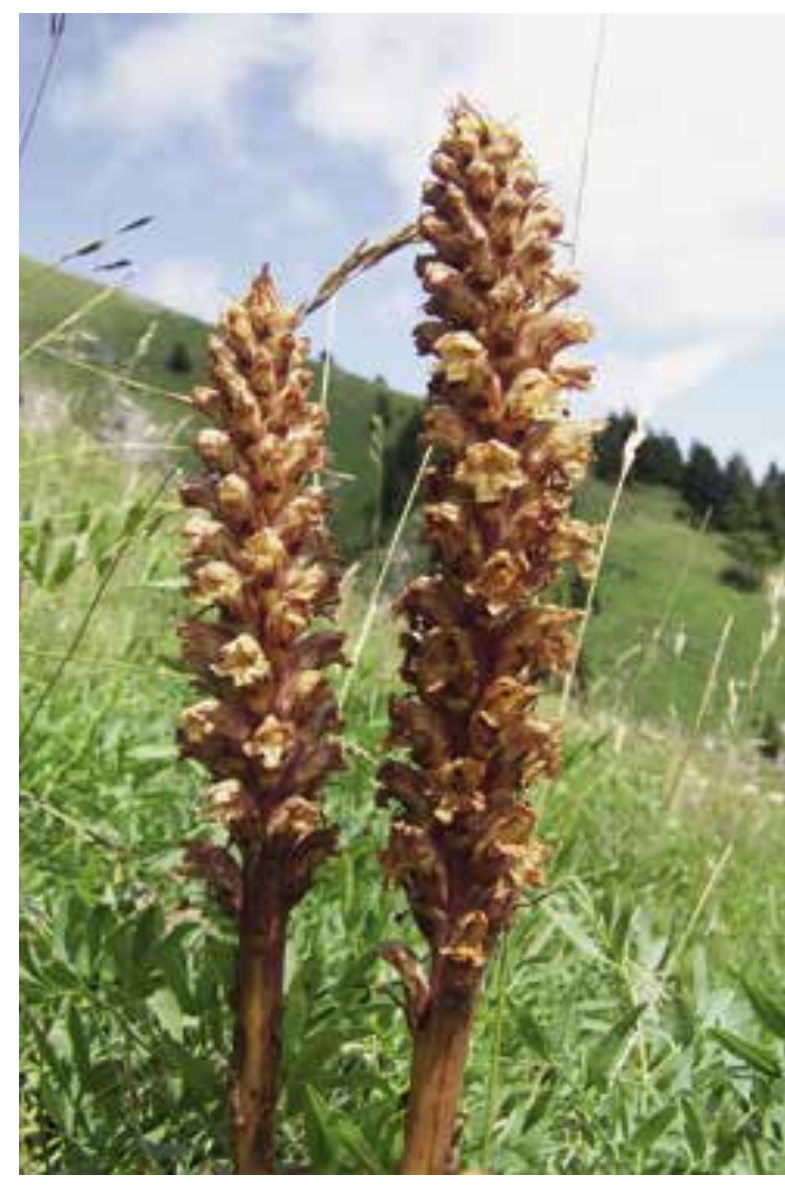

Amerika sind die Arten der Sektion Orobanche mit Saatgut eingeschleppt worden.

\section{Sommerwurz als Schädling in der Landwirtschaft}

Schon von jeher hat die Menschen der den Sommerwurzgewächsen eigene Parasitismus interessiert. Einer der ersten, der davon berichtete, war Theophrastos von Eresos $(371-287$ v. Chr.). Er beschreibt, wie die Sommerwurz ihr Opfer durch Umschlingen erwürgt. Ob er damit bereits die Pflanze meinte, die wir heute als Sommerwurz kennen, ist umstritten. Jedenfalls ist er mit für die Namensgebung verantwortlich: Orobanche leitet sich von grch. orobos (Kichererbse) und grch. anchein (würgen) ab. Im Laufe der Zeit erhielt die Sommerwurz weitere, mehr oder weniger schmeichelhafte Namen. Der Umstand, dass die Sommerwurz vor Kraft strotzt, während der Wirt vor sich hin siecht, ließ die Fantasie der Menschen ins Kraut schießen. „Wolf“, „Würger“, „Franzos“ - je nach Zeitgeschichte hatten die Menschen viele 
Schimpfnamen für die Pflanze, die, manchmal massenweise auftretend, ihre Ernte vernichtete. Manch ein Artikel in einer Gartenzeitung zeugt von starken Antipathien gegenüber Orobanche. Anfang des 20. Jahrhunderts gab es z. B. im Rheinland ein Gesetz, dass es unter (Geld)Strafe stellte, wenn man die Sommerwurz (in diesem Fall Orobanche minor) auf eigenem Grund und Boden zur Blüte kommen ließe. Orobanche ist in der Lage, ohne weiteres bis zu 300000 winzige Samen pro Pflanze zu erzeugen, die dann vom Wind ausgebreitet werden.

Auch heute beschäftigen sich viele der Landwirtschaft nahe stehende wissenschaftliche Institute mit der Frage, wie sich die SommerwurzPflanzen am effektivsten vernichten lassen, ohne die Kultur zu schädigen. Die Züchtung von resistenten Tomaten-, Kartoffel- und Sonnenblumenrassen ist genauso Gegenstand zahlreicher Publikationen wie die Entwicklung von die Sommerwurz schädigenden Phytoparasiten oder Herbiziden.

Untersucht ist, dass der Sommerwurz-Samen, wenn er nach seinem Flug vom Regen in den Boden gespült wurde, auf phytochemische Reize reagiert, die die vitale Wirtspflanze aussendet. Dann entwickelt sich ein wurzelähnliches Primärhaustorium, welches den Anschluss an die Wurzel des Wirtes sucht. Ist dies geschehen, kann die Sommerwurz über weitere Sekundärhaustorien ein ganzes Geflecht von Verbindungsleitungen zwischen sich und dem Wirt oder weiteren Wirten schaffen. Noch nicht abschließend geklärt ist die Frage, ob die Sommerwurz auch die Ruhephasen außerhalb der Blütezeit auf den Wirten überdauert.

Es gibt Sommerwurz-Arten (z. B. Orobanche minor, O. cernua, Phelipanche ramosa), die ihre Lebensweise auf eine Vielzahl von Wirten vieler Familien abgestimmt haben, also polyphag sind. Andere befallen nur wenige Arten einer oder mehrerer Familien, sie sind oligophag. Dies sind z. B. Orobanche lucorum, O. flava, O. hederae und $O$. laserpitii-sileris. Nur eine einzige Wirtsart befallen z. B. Orobanche salviae (auf Salvia glutinosa), $O$. artemisiae-campestris und Phelipanche bohemica (jeweils auf Artemisia campestris).
Bei der Bestimmung einer Sommerwurz kann man sich aber nicht auf die Bestimmung des Wirtes beschränken. Nicht immer oder eher selten ist die der Sommerwurz räumlich am nächsten stehende Pflanze ihr Wirt. Es kommt auch vor, dass die weiche SommerwurzStängelbasis in der Erde von anderen Wurzeln durchdrungen wird, ohne dass es zu einer WirtParasit-Beziehung kommt. Das Ausgraben einer Sommerwurz mit ihrem Wirt zur eindeutigen Bestimmung ist namentlich in steinigen Gebieten oft nicht erfolgreich und sollte ohnehin wegen deren Seltenheit die Ausnahme bleiben. Daher sind viele in der Literatur kursierende Wirtsangaben mit Vorsicht zu genießen. Es ist bislang nicht in einem einzigen Fall mit Sicherheit nachgewiesen worden, dass eine Sommerwurz auch einkeimblättrige Wirte befällt. Ebenso wenig ist ein Bastard verschiedener Orobanche- oder Phelipanche-Arten bekannt. Es existieren nur wenige unsichere Angaben hierzu.

\section{Sommerwurz in Hessen}

Einige Sommerwurz-Arten gehören zur Flora von Hessen. Die Ästige Sommerwurz (Phelipanche ramosa) kam früher besonders in Hanffeldern vor (z. B. bei Hanau, Okriftel), ist nun aber mit dem Niedergang des Hanfanbaus in Hessen ausgestorben. Man findet sie u.a. noch in Baden-Württemberg in der Nordbadischen Rheinebene (auf Tabak). Die Sand-Sommerwurz (Phelipanche arenaria) wurde für Hessen bereits 1794 von BorkHAUSEN erwähnt (Arheilgen bei Darmstadt). Hier sowie an anderen Stellen um Darmstadt und an der Bergstraße kommt sie heute noch vor, obwohl die Art sehr stark zurückgeht. Eine der wenigen Arten, die sich leicht ausbreiten, ist die Purpur-Sommerwurz (Phelipanche purpurea). Sie tritt zerstreut in Magerrasen auf, gern auch als Weinbaubegleiter an Wegrändern. In Hessen parasitiert sie vor allem auf Schafgarbe (Achillea millefoilium). Um Darmstadt kommt auf Sandböden hin und wieder auch die Weiße oder Quendelsommerwurz (Orobanche alba) vor. Ihre Wirte sind Lippenblütler, vor allem Thymian. Außer um Darmstadt kommt sie in Hessen auch in der 
Rhön und an der Landesgrenze zu Rheinhessen vor. Sie ist jedoch nicht nur an Sand gebunden und beispielsweise auch eine relativ verbreitete Art auf Kalk in den Alpen. Die ähnliche Netzoder Distel-Sommerwurz (Orobanche reticula$t a)$ ist in Hessen nur an der Landesgrenze zu Thüringen und Bayern in der Rhön und von der nordbadischen Rheinebene ausstrahlend bei Groß-Gerau zu finden. Sie parasitiert auf Distel-Arten in ruderal beeinflussten Staudenfluren und auf Brachen.

Die Nelken-Sommerwurz (Orobanche caryophyllacea) duftet leicht nach Gewürznelken. Ihre Wirte sind Rötegewächse (Galium, Asperula). In Hessen ist sie zerstreut und keinesfalls häufig. Ihr nahe verwandt, aber deutlich seltener ist die Gelbe Sommerwurz (Orobanche lutea). Sie gedeiht im Sandgebiet um Darmstadt, an der Bergstraße und in der Rheinebene, im Mainzer Sand, aber auch in Frankfurt. Sie befällt vor allem Luzerne (Medicago), jedoch nicht in Kulturen. Sehr selten finden wir am Südrand von Hessen bei Hemsbach die BitterkrautSommerwurz (Orobanche picridis) und die sehr ähnliche Kleine Sommerwurz (Orobanche minor). Erstere Art wächst vorzugsweise in lückigen Wiesen und auf Brachen auf Picris hieracioides. Letztere war vor etwa 200 Jahren eine Plage in rheinländischen Kleefeldern und ist heute, wahrscheinlich durch modernere Methoden der Saatgutreinigung, eher selten. Ihre Wirte sind verschiedene Leguminosen, besonders Klee, aber auch Vertreter viele anderer Familien. Beide Arten sind im Mittelmeergebiet deutlich häufiger anzutreffen. Orobanche minor wurde mit Saatgut auch nach Nord- und Südamerika, Südafrika und Australien verschleppt.

Die auch im Palmengarten wachsende EfeuSommerwurz (Orobanche hederae) dürfte in Hessen kein natürliches Areal haben. Sie parasitiert sie vor allem auf Efeu, aber auch auf anderen Vertretern der Araliengewächse. Ihre

Abb. 5 (oben): Orobanche alsatica, Bergstraße, Hemsbach (28.5.2011).

Abb. 6 (unten): Orobanche lutea, Frankfurt, Berger Hang (7.5.2011).
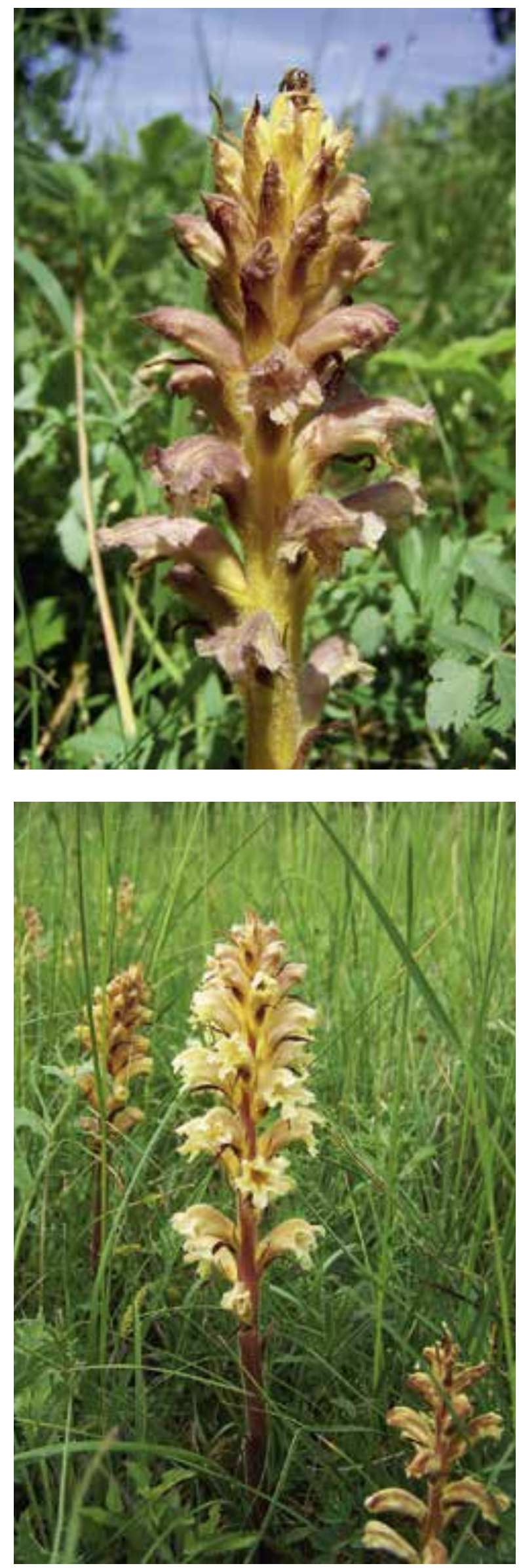
Heimat ist das Mittelmeergebiet, östlich bis an die Schwarzmeerküste. In klimatisch günstigen Gegenden, wie z. B. die vom Golfstrom geprägten Westküsten Englands und Irlands, wanderte sie ein. In Deutschland ist sie vor allem an alten Burganlagen, in Parks und Friedhöfen anzutreffen.

Die Elsässer Sommerwurz (Orobanche alsatica) wächst in ihrer typischen Varietät auch im Botanischen Garten Frankfurt. Sie parasitiert auf Hirschwurz (Cervaria rivini). Die var. libanotidis befällt Berg-Heilwurz (Seseli libanotis). Orobanche alsatica var. typica gibt es in Hessen nur am Südrand bei Hemsbach. Die var. libanotidis kommt in Nordhessen bei Bad SodenAllendorf vor und wurde von dort 1844 von Grisebach als Orobanche bartlingii erstmals beschrieben.

Die Hohe Sommerwurz (Orobanche elatior) wächst auf Centaurea scabiosa. In Hessen ist sie von der Bergstraße bei Hemsbach bekannt. $\mathrm{Ob}$ die Vorkommen bei Mainz, Fulda und in Rheinhessen noch bestehen, ist ungewiss. Die Ginster-Sommerwurz (Orobanche rapum-genistae) ist eine westeuropäische Art. Sie hat ihre östlichsten Vorkommen in NW-Hessen an der Landesgrenze zu Nordrhein-Westfalen. Sie bevorzugt sandige Standorte und gilt entsprechend ihrem Wirt Cytisus scoparius als Charakterart der Besenginsterheiden. Die Zierliche oder Blutrote Sommerwurz (Orobanche gracilis) wurde in Gießen (1950 Halde am Bergwerk und 1971 Gießen-Leigestern) gefunden. Sie dürfte dort mit Graseinsaaten eingeschleppt worden sein. Ihre Wirtspflanzen sind Leguminosen (bes. Hornklee). In den Alpen ist sie eine Charakterart subalpiner Rasen über Kalk.

\section{Schlussbemerkung}

Sommerwurz-Arten sind nicht einfach zu bestimmen, selbst Spezialisten haben dabei manchmal Schwierigkeiten. Es ist wichtig, zur Bestimmung voll erblühte Pflanzen heranzuziehen, da nur an ihnen die wesentlichen Merkmale charakteristisch entwickelt sind. Ebenfalls wichtig ist es, sich die Merkmale zu notieren, die an gesammelten und getrockneten Pflanzen nicht mehr sichtbar sind (z. B. Farbmerkmale von Stängel und Krone, bes. auch die Narbenfarbe). Schließlich sollten die benachbarten oder in der Nähe stehenden Pflanzen als potentielle Wirte bestimmt und notiert werden. Ein derzeit im Aufbau befindliches Wiki-Projekt (http://orowiki.org) soll vorhandenes Wissen über die Orobanchaceae sammeln und künftig bei der sicheren Bestimmung dieser kritischen Gruppe helfen. Denjenigen, die sich vertiefter mit Orobanchen beschäftigen möchten und brauchbare Bestimmungsschlüssel und Artbeschreibungen suchen, sind die unten genannten Publikationen zu empfehlen.

\section{Dank}

Großer Dank gilt den Mitarbeiterinen und Mitarbeitern des Botanischen Gartens Frankfurt am Main, besonders auch ANDreas KöNIG und Manfred Wessel, für die freundliche Unterstützung.

\section{Literatur}

Kreutz, C. A. J. 1995: Orobanche. Die Sommerwurzarten Europas. Mittel- und Nordeuropa. Natuurhistorisch Genootschap in Limburg. - Limburg.

Uhlich, H., Pusch, J. Barthel, K.-J.1995: Die Sommerwurzarten Europas. Neue Brehm Bücherei, Bd. 618. - Magdeburg.

Pusch, J. \& Günther, K. - F. 2009: Orobanchaceae. - In: Hegi, G. (Hrsg.) Illustrierte Flora von Mitteleuropa. Band VI. Teil 1A. - Jena.

Beck von Mannagetta, G. Ritter 1890: Monographie der Gattung Orobanche. - Kassel.

Beck von Mannagetta, G. Ritter 1930: Orobanchaceae. - In: Engler, A. (Hrsg.): Das Pflanzenreich IV. - Leipzig. 